\title{
35. COMMISSION DE LA CONSTITUTION DES ETOILES
}

Président: M. T. G. Cowling.

Membres: MM. Bethe, Biermann, Mme Bondi, MM. Bondi, Chandrasekhar, Dingens, Epstein, Ferraro, Gamow, Gratton, Hoyle, Martin Johnson, S. A. Kaplan, Keller, Kozyrev, Kothari, Lebedinsky, Ledoux, Lemaître, Lyttleton, Mme Masevich, MM. McCrea, McVittie, G. Münch, Odgers, Osawa, Qvist, Ramsey, Randers, Reiz, Rosseland, Russell, Schatzman, Schwarzschild, Severny, Strömgren, Sweet, R. N. Thomas, Tiercy†, Tuominen, von Weizsäcker, von Zeipel, Wasiutynski, Williamson.

In I953 took place a conference on the dynamics of the interstellar medium at Cambridge, and the Liège colloquium on nuclear processes in the heavenly bodies. The discussions of the first of these were mainly on subjects outside the province of this Commission, but included a discussion on accretion. The papers presented to the Liège colloquium $(x)$ were largely on stellar structure and composition. A number of them are referred to below; others may be of interest to members of the commission.

\section{Physical Fundamentals}

In the past few years the reaction rates for the $\mathrm{C}-\mathrm{N}$ and $\mathrm{P}-\mathrm{P}$ processes for stellar energy-generation have become more accurately known $(2,3)$. An authoritative summary of the results is given in a paper by Fowler (4). They indicate that stars earlier in spectral type than the Sun generate their energy mainly by the $\mathrm{C}-\mathrm{N}$ cycle: the Sun and dwarfs of later spectral type chiefly use the $\mathbf{P}-\mathbf{P}$ reaction. Bosman-Crespin, Fowler and Humblet have calculated numerical values for both reactions for temperatures ranging from I to 50 million deg. $\mathrm{K}(5)$.

Energy-generation in collapsing helium stars was considered by Salpeter (6), who showed that at temperatures above $10^{8} \mathrm{deg}$. $\mathrm{K}$ helium atoms would readily build into carbon, oxygen and perhaps higher nuclei. A similar suggestion had been made independently by Öpik ( $)$.

The problem of the origin of the elements is one whose place is midway between astrophysics and theoretical physics. Here it is sufficient to draw attention to three comprehensive surveys of the problem, by Podolanski and ter Haar (8), by Gurevich (9) and by Hoyle (xo).

Studies of the structure of special stars have shown that existing data on the opacity of stellar material are seriously inadequate, particularly in the disregard of opacity due to free-free transitions of hydrogen. The free-free opacity was taken into account in solar models by Naur (r1) and Epstein and Motz (r2). Detailed calculations of the free-free opacity have been made by Tsao(13), by Zirin (14) and by Kulsrud (x5); their work was done independently and a comparison of their basic assumptions and results has not yet been given. Calculations of the total opacity of stellar material have been made by Keller and Meyerott (56) for a number of assumed compositions at temperatures ranging from $10^{5}$ to $2 \times \mathrm{IO}^{7}$ degrees. These calculations take into account electron scattering as well as bound-free and free-free opacity. Not all the results have as yet been published, but the authors hope to be able to supply mimeograph tables on request.

Finally, the work of Chandrasekhar (zy) on the effects of Coriolis forces or a magnetic field on thermal instability should be mentioned. This work is not immediately applicable to stellar structure; it uses boundary conditions appropriate rather to laboratory experiments, and is concerned with marginal stability, not with instability. But any assistance in elucidating the unsolved problems of turbulence in rotating or magnetic stars is valuable. Gurevich ( 18 ) has emphasized the important effects on stellar turbulence when the rotational and magnetic axes do not coincide. 


\section{MOdels of Stationary Stars}

\section{General}

Sedov(rg) examined the mass-luminosity and mass-radius relations obtained by assuming the molecular weight, generation of energy and opacity to be proportional to powers of the density and temperature. Emin-Zade (20) constructed a model of a dense star with a convective core and a radiative envelope with opacity proportional to $T^{\mathbf{2}}$. This model, which gives high central densities and temperatures, is not applicable to any type of star possessing thermonuclear energy sources. Severny (2I) investigated models with any energy sources concentrated towards the centre, and calculated the hydrogen and helium content for a number of main sequence stars. Masevich and Dokuchaeva (2z) studied a model with a convective core and an envelope with opacity due to scattering only, and showed that such a model cannot explain the stars actually existing.

Naur and Osterbrock (23) discussed the condition for a star to possess a convective core. Assuming generation and opacity laws of the forms $\epsilon=\epsilon_{0} \rho^{\delta} T^{\nu}, \kappa=\kappa_{0} \rho^{1-\alpha} T^{-\beta}$, a convective core cannot exist unless $6 \nu+9 \delta>I 5 \alpha+$ Io $\beta$; this condition excludes red dwarfs. Ledoux (24) pointed out that this criterion is really a criterion for the instability to be maximum at the centre, and suggested that in some cases convective and radiative zones might alternate.

Levee (25) considered a model deriving its energy from gravitational contraction, with opacity given by Kramers' law. He found a time-scale $5 \times 10^{7}$ years for the Sun on this model.

\section{Models with discontinuities}

Schwarzschild and his collaborators (26) have continued their work on models with a discontinuity in the molecular weight $\mu$. Oke and Schwarzschild conducted an investigation along now familiar lines for explaining red giants, assuming a convective core and a discontinuity in $\mu$ whose position was varied. They derived satisfactorily large radii, but masses which were perhaps rather low. Sandage and Schwarzschild considered stars with exhausted cores, no longer able to exist in equilibrium. As the core contracts, the envelope expands, and the star moves to the right across the Hertzsprung-Russell diagram. The models cover the red-giant range. Schwarzschild, Rabinowitz and Härm considered inhomogeneous models with a partially degenerate exhausted core, the discontinuity being at the boundary of the core. If about one-third of the mass is in the core, one again gets radii characteristic of red giants. An extensive tabulation cf numerical integrations has been prepared by Härm and Schwarzschild (27).

Roy (28) has considered inodels with a discontinuity in $\mu$ at the boundary of a convective core, using first Kramers's-law opacity and then electron-scattering in the outer envelope. Reznikov (29) studied a model with an isothermal non-degenerate core and a radiative envelope of different $\mu$, again using pure absorption and pure scattering in the envelope.

Mme Dumézil-Curien (30) has considered a model for red giants with an extensive convective outer region as well as the usual core of high $\mu$. Conditions near the surface may have to be taken seriously into account in future work.

Mme Bosman-Crespin (3I) has considered models for hot (O-B) stars with and without a discontinuity in $\mu$ between the convective core and the radiative envelope. Without a discontinuity the abundances required for helium and heavy elements are greater than one would expect; with a discontinuity the proportion of helium is reduced to about one-sixth by weight, but the proportion of heavy elements remains a little high.

\section{Degeneracy: white dwarfs}

V. S. Sorokin (32) investigated in detail the equilibrium of isothermal gas spheres of different degrees of degeneracy, taking into account the pressure of the ions as well as that due to the degenerate electrons. Sorokin also considers the stability of such spheres 
for certain rather artificial displacements. Kaplan and Klimishin (33) improved earlier determinations of the parameters of degenerate configurations. Rudkjøbing (34) developed Chandrasekhar's theory of white dwarfs, taking into account relativistic spin-orbit interaction; this decreases the radius corresponding to a given mass. Bandyopadhyay (3s) extended the theory of the Chandrasekhar limit to stars with angular velocities up to a thousand times the earth's.

Schatzman (36) estimated the internal temperature of white dwarfs for balance between generation and emission of energy, assuming them to consist of a core of heavy elements with a hydrogen mantle. However, Mestel (37) showed that white dwarfs need no subatomic energy sources; they can provide their radiation from their heat energy. If they should acquire such energy sources, they might explode as supernovae. If there is balance of generation and emission of energy, they are, thermally, in a state of unstable equilibrium.

\section{Models for special stars}

A number of models for the Sun have been calculated. Epstein (38) calculated a model taking account of both $\mathrm{P}-\mathrm{P}$ and $\mathrm{C}-\mathrm{N}$ generation of energy; however, his assumed rate for the $\mathrm{P}-\mathrm{P}$ reaction was incorrect and no account was taken of free-free opacity. Epstein and Motz (39), in the first of two papers, corrected the error in the $\mathrm{P}-\mathrm{P}$ reaction rate; they found it barely possible, even assuming zero helium content, to construct a possible model. In the second, free-free opacity was also taken into account; the percentage of helium was then found to be 6.7 , and the central density and temperature were $98 \mathrm{gr} . / \mathrm{cm}^{3}$ and nearly $\mathrm{I} 3$ million degrees. A convective core extends through $8 \%$ of the solar radius.

In a paper based on similar assumptions Naur (40) found a rather higher central temperatureand a helium percentage about 25 . Frank-Kamenczky (4r), in a similar calculation, found a rather lower central temperature and I $8 \cdot \mathrm{I} \%$ helium. Further improvements in the model appear to depend on improved calculations of opacity and energy generation. So far the models have assumed uniform composition, but Ledoux is working on a model with an increased fraction of helium near the centre. Biermann (42) has pointed out that the present reduced central temperatures have brought matters near to the point where degeneracy has to be taken into account.

Aller et al. (43) found that $\mathrm{P}-\mathrm{P}$ generation should give red dwarfs too high a luminosity. Osterbrock (44), following an earlier suggestion by Strömgren, showed that the difficulty was removed in the case of dwarfs like Castor $\mathrm{C}$ by an extensive hydrogen convection zone, whose depth is about $30 \%$ of the stellar radii. A discrepancy of unknown origin still appears to persist for dwarfs of still later type, like $\mathrm{Kr} 60 \mathrm{~A}$. The latter star was considered by Emin-Zade (45) on a model with an isothermal core and an opacity proportional to $T^{-\mathbf{2}}$, using either $\mathrm{C}-\mathrm{N}$ or $\mathrm{P}-\mathrm{P}$ generation.

Mme Soushkina (46) has calculated a sequence of models for giant stars, with partially degenerate isothermal cores. The model for Capella, based on the $\mathrm{C}-\mathrm{N}$ cycle, and assuming negligible helium content, gave $40 \%$ hydrogen.

Epstein and his collaborators are now engaged on a model for Procyon, taking into account a certain amount of variation of $\mu$ between central and outer regions. Kushwaha, at Princeton, is deriving models for B and early A stars, and hopes to be able to follow their early evolution.

Reiz has attempted to explain the location in the H-R diagram of Population II high-velocity sub-dwarfs of type $\mathrm{F}$ in terms of a stellar model built on the assumption of negligible heavy-element content. This assumption implies that the opacity is due mainly to free-free transitions and that the energy production is by the proton-proton reaction. The configurations were found to correspond roughly to the region of the H-R diagram where the sub-dwarfs are located, but there were some deviations from actual subdwarfs(II2). 


\section{Rotation, Mixing CurRents, etc.}

Parijsky (47) showed that although the Sun might possess a rapidly rotating core, its angular momentum could not exceed $2 \%$ of that of the solar system. Thus such a core would not remove the angular momentum difficulty of Laplace-type cosmogonic theories.

Sweet and Roy (48) determined the first-order effect of uniform rotation on the structure of a star generating energy by the $\mathrm{C}-\mathrm{N}$ cycle. They found that observed rotations can produce a spread in luminosity up to half a magnitude for Be-type stars.

Motz (49) compared the radius of gyration $H$ with the apsidal-motion constant $k$ for a large number of stellar models. He found that, save for certain exceptional models, an approximately linear relation connects $\log k$ and $\log (H / R)$, where $R$ is the star's radius.

Mestel (50) showed that mixing currents due to rotation can be choked back by the distribution of molecular weight which they themselves set up. Little mixing by such currents is possible in any uniformly rotating star. The work of Greenstein and his collaborators (5x) on the abundance of $\mathrm{Li}, \mathrm{Be}$ and $\mathrm{C}^{13}$ in the Sun has shown that there is no appreciable mixing between surface layers and a depth where the temperature exceeds 3.5 million degrees. In unpublished work with J. B. Oke, Greenstein has also found that angular momentum considerations are consistent with $\mathrm{A}, \mathrm{F}$ and $\mathrm{G}$ type giants having originated as rapidly rotating $B$ and $A$ stars, and having developed exhausted cores of higher molecular weight. If this has occurred, rapid rotation has not resulted in substantial mixing.

\section{Mass-luminosity-radius relations}

Inferences drawn from the observed relations between the mass $M$, luminosity $L$ and radius $R$ have been surprisingly varied. Bondi (52) suggests that, contrary to the assertion that transition from $\mathrm{C}-\mathrm{N}$ to $\mathrm{P}-\mathrm{P}$ generation takes place at a spectral class just earlier than the Sun's, there is no irregularity in the observed relations until type Ko. Parenago, on the other hand (s3, 54), finds a definite discontinuity in the $M, L, R$ relations about spectral class $\mathrm{G}_{5}$. Kozyrev (55) deduced from the $M, L, R$ relations that thermonuclear relations are not the source of stellar energy.

Gurevich (56) suggested that the $\log L, \log M$ curve is S-shaped, with slopes $2 \cdot 3,5 \cdot 7$ and 3 in the ranges $M<M_{\odot}, M_{\odot}<M<2 \cdot 7 M_{\odot}$ and $M>2 \cdot 7 M_{\odot}$ respectively. This he attributed to changes in the absorption law.

Frank-Kamenczky (57) studied the mass-luminosity law for pure hydrogen stars, taking into account both absorption and scattering. He obtained a satisfactory agreement with real stars in the range $A-K$.

Kurth (58) used the $M-L$ and $M-R$ relations to identify homologous sequences among the stars.

\section{Computation}

A number of workers have noted the possibility of using electronic computers for integrating the equations of stellar structure. Hoyle suggests, indeed, that 'enough is probably known now about the problems of stellar structure to drop hand computations entirely in favour of the big electrical machines'.

The only paper known to me which describes work done on an electronic computer is one by Osterbrock (59), quoted above. But similar work has been begun, or is about to be begun, by Kopal at Manchester, by Hoyle at Cambridge, by collaborators of Qvist at Stockholm, and by Reiz, as well as probably two or three others of whom I have heard indirectly. Numerical results can be produced so rapidly by such computers that the chief problems may become their control and co-ordination and the dissemination of results. 


\section{NON-STATIONARY STARS}

\section{Novae}

Two problems have been studied in the theory of novae. These are the generation of nuclear explosions in a star, and the propagation of an initial impulse outward as a shock wave.

Schatzman (60) considered the possibility that nova outbursts are due to explosive reactions involving $\mathrm{He}^{3}$. Gurevich (6r) studied their possible origin as a result of accretion of deuterium-rich material, supernovae occurring when this material is absorbed sufficiently fast. For ordinary novae nuclear explosions might occur in the outer layers of a star. The difficulty in such theories is to ensure that the reaction is explosive (see Mestel (62)).

Schatzman (63) has considered afresh recurrent nova explosions. His formula for their recurrence has been given an observational check by Mlle Zuckermann (64).

Kopal (65) considered the propagation of spherical shock waves from the centre of a self-gravitating gas sphere. The interest of his paper is mainly mathematical. Sedov(ri3) showed that waves generated by a central explosion could either blow off the surface layers or scatter the whole star.

Mme Dumézil-Curien (66) showed that a compression wave passing up into a stellar atmosphere could generate an acoustic radiation pressure greater than gravity.

\section{Pulsations and stability}

One important result, recognized by a number of workers (e.g. Ledoux (67)), is that the increase in radius of classical cepheids resulting from their increase in absolute luminosity (Baade-Mineur) is able to produce agreement between the pulsation theory and observation so far as periods are concerned.

Ledoux (68) has investigated the effect on radial pulsations of a decrease in the effective polytropic index in the external regions. He has also shown how to apply the virial theorem to non-linear radial oscillations (69), though his results still are unable to explain the observed asymmetry.

Schatzman $(70)$ considered the temperature-dependence of thermonuclear reactions in relation to vibrational stability. Frank-Kamenczky (7x) developed a theory of central pulsations of variable stars based on ideas analogous to those of Schatzman. From an approximate form of the equation of motion for finite oscillations he deduced a theoretical period-amplitude relation similar to the empirical relation found by Parenago and Kukarkin.

Zhevakin (72), on the other hand, developed a theory of peripheral pulsations, according to which the oscillations are maintained by the zone of critical second ionization of helium. Keller is investigating the effect of anomalies in the opacity law on cepheid pulsation.

Huang (73) studied the application of the Ritz method to radial oscillations. A number of other mathematical studies of radial oscillations and stability have also been made (74), but owing to the artificiality of the stellar models the interest of this work is mainly formal.

\section{Magnetic stars}

Babcock's discovery of magnetic stars, and the suggestion that their frequent variability might be due to magnetically controlled oscillations, has stimulated several papers on the equilibrium, stability and pulsations of such stars. Because of mathematical difficulty, a uniform liquid star has often been assumed. Investigations have not yet gone sufficiently far to indicate which of the results depend on the model, and which are applicable to real stars.

Miss Gjellestad (75) has considered the equilibrium distortion of a liquid sphere in its 
own magnetic field, and in a uniform external magnetic field, using an energy method. Her work in the former case was refined by Ferraro (76), who used the detailed equations of equilibrium. Both workers found that appreciable distortion can occur only if the magnetic energy is comparable with the gravitational. Miss Gjellestad's formulae indicated that in a uniform external field the sphere would become prolate.

Miss Gjellestad (77) considered the gravitational oscillations of a liquid sphere, as perturbed by a magnetic field. Ferraro and Memory (78) considered a liquid sphere in the presence of a radial (unipolar) magnetic field, and concentrated their attention on the oscillations in which the magnetic field is of dominant importance. They found that such oscillations were far too slow for an oscillation to be a likely cause of the observed variability of magnetic stars. Plumpton and Ferraro (79), by a more refined argument, were able only to narrow the discrepancy a little.

Cowling (80) attempted a rough general discussion of magnetic oscillations for a stable star (a uniform liquid is neutral for internal displacements). He found that gravity now had so strong an effect that oscillations slow enough to represent observed stars were hard to produce. He also indicated that oscillations were only in rare circumstances able to produce a reversing field. His conclusions in the latter connexion were given a detailed confirmation by Sweet (8I).

Chandrasekhar and Fermi (82) considered a number of problems of gravitational stability in the presence of a magnetic field. In particular they suggested that the presence of a sufficiently large magnetic field might destroy a star's stability. This conclusion received confirmation in a paper by Chandrasekhar and Limber $\left(8_{3}\right)$ on radial pulsations. An illustrative cylindrical problem was discussed by Lyttkens (84).

Stoyko (85) discussed a possible connexion between magnetic variability and variations in angular velocity.

Wrubel (86) calculated times of decay for the principal and other modes of a magnetic field in a star. Using Schwarzschild's temperature distribution, he found a time of decay $4 \times 10^{9}$ years for the principal mode in the Sun.

\section{COSMOgONY AND STARS}

\section{Formation of stars}

Hoyle (87) discussed the formation of galaxies, groups of stars and stars from tenuous clouds by a process of slow contraction of the parts alternating with periods of gravitational instability and fission. He considered in particular differences in origin between populations I and II. Lebedinsky (88) also considered the formation of stars from diffuse matter, using a criterion according to which stars with masses exceeding a certain value should spontaneously divide, and taking into account mutual interaction between stars and diffuse matter. Krat (89) advanced a hypothesis of the origin of stars, stellar systems and planets from comet-like prestellar bodies with high contents of light elements, existing comets being regarded as relics of these bodies. Stellar formation occurs after an intermediate phase of a gas-dust nebula.

Ambartsumian (9o) has argued in a reverse direction. He starts with the known expanding stellar associations, whose positive total energy cannot be explained by stellar formation by gravitational instability. He deduces that they arise from 'protostars' of very great mass, consisting of matter with abnormal properties. An explosive reaction in this 'prestellar' material generates the expanding association.

Ledoux has considered gravitational stability in a flat isothermal nebula, with reference to stellar formation ( $9 \mathrm{r})$.

Gurevich and Lebedinsky (92) concluded from a study of the balance with diffuse matter in the Galaxy that at present stars are originating in the Galaxy mainly as dwarfs. The probability of transformation of dwarfs into giants was estimated. 


\section{Emission and accretion of matter}

Parenago (93) deduced a theoretical luminosity distribution, assuming that newly-born stars enter the main sequence in the region of the spectral class $\mathrm{O}$ and advance along it as they evolve with diminishing mass. The result agrees fairly well with observation in the range of absolute magnitudes from $-4 \mathrm{~m}$. to $+5 \mathrm{~m}$. Kaplan (94), assuming that the young stars enter the main sequence at different places, determined from the observed luminosity distribution the probable properties of the young stars. He found that approximately $80 \%$ of the young stars should have masses in the range $6-18 M \odot$ and spectral types between $\mathrm{O}_{9}$ and $\mathrm{B}_{4}$.

Mustel (95) studied the different factors which may cause corpuscular emission from the Sun. Schatzman (96) suggested that the instability of progressive waves moving outwards might lead to ejection of matter from stars.

Bondi considered spherically symmetric accretion by stars (97). He found that the velocity of sound in the accreted cloud at large distances was the main factor limiting accretion, this taking the place of the velocity of a star through a cloud in earlier theory. McCrea and Dodd $(98,99,100)$, in a number of papers, considered accretion by a star moving slowly through a cloud. They showed that the braking effect of the cloud could destroy the motion of the star relative to it, so that spherically symmetric accretion could follow, leading to the formation of a large star in a few million years. They have also considered the production of groups of bright stars, and binary stars.

A number of papers on accretion were presented to the Cambridge colloquium on the dynamics of interstellar gas. Some criticisms of accretion were based on the neglect of heating effects due to the radiation of the accreting star. Schatzman (ror) claimed to show that in all actual physical contexts accretion is certainly of negligible importance.

Mestel has considered the heating effects of radiation near an accreting star (roz). He finds that this can be large, but does not altogether prevent rapid accretion in a sufficiently dense cloud.

\section{Stellar evolution}

A general review of the present state of theories of stellar evolution has been given by Strömgren (ro3). This deals with theories of formation, stellar models, evolutionary changes in composition, and deductions from the $H-R$ diagram.

Evolutionary changes in composition are in effect the justification of many papers noted above, like those on red giants or mixing in stars. Such changes as are due to nuclear processes have been discussed by Greenstein (104). He lists the following groups of stars as apparently showing the influence of nuclear reactions now or in the past; Wolf-Rayet stars, helium and carbon stars deficient in hydrogen, hot subdwarfs, white dwarfs, high-velocity stars, and S stars, especially those showing the presence of technetium. This is an interesting list, each item of which provides a challenge to students of stellarstructure. However, Struve (ro5), in a study of subgiants, emphasized that an increased molecular weight might be due to the process of formation rather than evolution.

The early evolutionary phases of very heavy stars with pure electron-scattering have been followed in detail by Tayler (ro6). He finds that the evolutionary track turns sharply off the main sequence.

Mme Masevich (ro7) has studied evolutionary tracks for main-sequence stars assuming either constant mass or a steady loss of mass as given by Parenago's theory. She considers several stellar models, assuming in turn complete mixing and no mixing between core and envelope. She obtains models covering the subgiant region as well as the main sequence.

Schatzman (ro8) has considered a number of problems related to the formation and evolution of stars, in particular their kinematic and rotational properties. He suggests that some hot cluster stars may be young stars not yet having attained a steady state.

Some information on evolutionary tracks can be derived by a study of groups in the $H-R$ diagram. Sandage (rog) has attempted to combine such information for the clusters 
$\mathrm{M}_{3}$ and M67 with general results in the theory of stellar structure. He suggests that these are old clusters: the giants (type II) in them are very undermassive compared with type I stars of the same luminosity, and left the main sequence $5 \times 10^{9}$ years ago. Evolutionary tracks in $\mathrm{M} 67$ appear to differ from those in $\mathrm{M}_{3}$, perhaps because of greater metal abundances in M67. A comparison of results for different clusters suggests that evolutionary tracks in the $H-R$ diagram are nearly horizontal for very massive stars, but curve upward for less massive ones.

Reddish (Iо) similarly makes deductions from the $H-R$ diagram, in particular from the absence of a Hertzsprung gap for population II stars. He suggests that the Hertzsprung gap for population I results from rapid evolution as a star's convective core, exhausted of hydrogen, rapidly contracts. He supposes population II to have no convective cores; population I clusters show larger Hertzsprung gaps if composed of more massive stars, with a greater fraction of their mass in the cores.

Hoyle and Schwarzschild have studied the theoretical course of development of population II stars(rir). Their models show the passage from the main sequence to the giant branch on the $H-R$ diagram, and then return to a horizontal branch.

\section{RECOMMENDATION}

The Cosmogony Committee of the Astronomical Council of the U.S.S.R. Academy of Sciences states:

The time is now ripe for the establishment of a commission to co-ordinate cosmogonical work on the origin and evolution of planets, stars, and stellar systems, as well as papers in which problems of cosmogony are considered.

\section{REFERENCES}

(I) 'Les Processus Nucléaires dans les Astres' (report of the fifth Liège Colloquium). Mem. Soc. Roy. Sci. Liege, vol. r4, 1954.

(2) E. A. Frieman and L. Motz, Phys. Rev. 89, 648, 1953.

(3) E. E. Salpeter, Phys. Rev. 88, 547, 1952;Ap.J. I16, 649, 1952.

(4) W. A. Fowler, Lg. 88.

(5) D. Bosman-Crespin, W. A. Fowler and J. Humblet, Bull. Soc. Roy. Sci. Liège, No. 9-1o, 327, I954.

(6) E. E. Salpeter, $A p . J .115,326,1952$.

(7) E. J. Öpik, Lg. I87.

(8) J. Podolanski and D. ter Haar, Lg. I9.

(9) L. E. Gurevich, in Problems of Cosmogony (Moscow, I954), 2, p. I5I.

(Io) F. Hoyle, $A p . J$. Supplements, no. 5, I954.

(I I) P. Naur, $A p$. J. r19, 365, I954.

(12) I. Epstein and L. Motz, $A p . J$. rI7, 3I I, 1953; 120, I56, I954.

(13) H. T. H. Tsao, $A p . J$. I19, 70, I954.

(14) H. Zirin, $A p . J$. 119, 371, 1954.

(15) R. M. Kulsrud, $A p . J$. 119, 386, 1954.

(I6) G. Keller and R. E. Meyerott, Argonne Nat. Lab. Reps, nos. $477 \mathrm{I}$ and 4856 , 1952.

(r7) S. Chandrasekhar, Proc. Roy. Soc. A, 217, 306, I953; J. Rat. Mech. Anal. 3, I8I, I954; Phil. Mag. 43, 501, 1952.

(18) L. E. Gurevich, in Problems of Cosmogony, 2, I5I.

(19) L. I. Sedov, C.R. Acad. Sci. U.R.S.S. 94, 643, 1954.

(20) T. A. Emin-Zade, Astr. J. U.S.S.R. 30, 64, 1953.

$\ddagger$ This report will be referred to as $L g$. 
(2I) A. B. Severny, Astr. J. U.S.S.R. 31, 362, 1954.

(22) A. G. Masevich and O. D. Dokuchaeva, Astr. J. U.S.S.R. 29, 526, 1952.

(23) P. Naur and D. E. Osterbrock, $A p . J .117,306,1953$.

(24) P. Ledoux, C.R. Acad. Sci. Paris, 236, 238I, I953; Lg. 200.

(25) R. D. Levee, $A p . J$. 117, 200, I953.

(26) J. B. Oke and M. Schwarzschild, $A p . J$. Ir6, 317, r952. A. R. Sandage and M. Schwarzschild, $A p . J$. 116, 463, 1952. M. Schwarzschild, I. Rabinowitz and A. Härm, $A p . J$. I18, 326, 1953. A. Härm and M. Schwarzschild, $A P$. J. I21, 558, I955.

(27) A. Härm and M. Schwarrschild, $A p$. J. Suppl. no. I I, I954.

(28) A. E. Roy, Mon. Not. R. Astr. Soc. 112, 484, 1952; Lg. 139.

(29) A. O. Reznikov, Astr. J. U.S.S.R. 3I, 6o, 1954.

(30) P. Dumézil-Curien, Ann. Astrophys. 17, 197, 1954.

(3I) D. Bosman-Crespin, Ann. Astrophys. 17, 263, I954.

(32) V. S. Sorokin, Astr. J. U.S.S.R. 29, 25, 1952.

(33) S. A. Kaplan and I. A. Klimishin, Lvov Obs. Circ. no. 27, p. 17, 1953.

(34) M. Rudkjøbing, K. Danske Vidensk. Selsk., Mat.-fys. Medd. 27, no. 5, 1952.

(35) G. Bandyopadhyay, Bull. Calcutta Math. Soc. 44, 89, 1952.

(36) E. Schatzman, Ann. Astrophys. 15, 36I, 1952.

(37) L. Mestel, Mon. Not. R. Astr. Soc. Ir2, $5^{83}$ and 598, 1952.

(38) I. Epstein, $A p . J$. I14, 438, r95 I.

(39) I. Epstein and L. Motz, $A p . J$. II7, 31 I, 1953; 120, 156, 1954.

(40) P. Naur, $A p . J$. Ir9, 365, 1954.

(4I) D. A. Frank-Kamenczky, Astr. J. U.S.S.R. 32, I 39 and 326, 1955.

(42) L. Biermann, Lg. I3o.

(43) L. H. Aller et al., Ap. J. Ir5, 328, 1952.

(44) D. E. Osterbrock, $A p . J .118,529,1953$.

(45) T. A. Emin-Zade, Astr. J. U.S.S.R. 30, 64, 1953.

(46) E. I. Soushkina, Astr. J. U.S.S.R. 30, 180, 1953.

(47) N. N. Parijsky, in Problems of Cosmogony, 4, p. 6.

(48) P. A. Sweet and E. E. Roy, Mon. Not. R. Astr. Soc. 143, 7or, 1953.

(49) L. Motz, $A p . J$. 115, 562, 1952; 118, 147, 1953.

(50) L. Mestel, Mon. Not. R. Astr. Soc. I13, 7 I6, I953.

(5I) J. L. Greenstein, R. S. Richardson and M. Schwarzschild, Proc. Astr. Soc. Pacif. 62, I 5, I950; J. L. Greenstein and R. S. Richardson, $A p . J .113,536$, 195I; J. L. Greenstein and E. Tandberg Hansen, $A p . J$. II9, I I3, I9.54.

(52) H. Bondi, Lg. I I2.

(53) P. P. Parenago, in Problems of Cosmogony, 2, p. $26 \mathrm{r}$.

(54) P. P. Parenago and A. G. Masevich, Astr. J. U.S.S.R. 2i, 137 and 202, 1950.

(55) N. A. Kozyrev, Crim. Obs. Publ. 6, 54, I95I.

(56) L. E. Gurevich, C.R. Acad. Sci. U.R.S.S. 92, 499, 1952.

(57) D. A. Frank-Kamenczky, Astr. J.U.S.S.R. 31, 327, I954.

(58) R. Kurth, $Z$. Ap. 32, II, I953.

(59) D. E. Osterbrock, $A p . J$. 118, 529, 1953.

(6o) E. Schatzman, Ann. Astrophys. 14, 294 and 305, I95I.

(6I) L. E. Gurevich, in Problems of Cosmogony, 3, p. 94; Publ. $4^{\text {th }}$ Cosmogonic Conference, I954 (in the Press).

(62) L. Mestel, Mon. Not. R. Astr. Soc. Ir2, $5^{83}$ and 598, 1952.

(63) E. Schatzman, Ann. Astrophys. 17, 305, 1954.

(64) M. C. Zuckermann, Ann. Astrophys. I7, 243, 1954.

(65) Z. Kopal, $A p . J$. 120, I 59, 1954.

(66) P. Dumézil-Curien, C.R. Acad. Sci. Paris, 235, 1364, 1952.

(67) P. Ledoux, Ciel et Terre, 68, nos. I-2 and 3-4, I953; P. Grandjean and P. Ledoux, Ann. Astrophys. 17, 161, 1954.

(68) P. Ledoux, Bull. Roy. Soc. Sci. Liege, 2r, 408, 1952.

(69) P. Ledoux, Bull. Acad. Roy. Belg. 38, 352, $195^{2}$. 
(70) E. Schatzman, Ann. Astrophys. 16, I62, 1953.

(7I) D. A. Frank-Kamenczky, C.R. Acad. Sci. U.R.S.S. 77, 385, 1951; 80, 185, 1951;86, $808,1952$.

(72) S. N. Zhevakin, Astr. J. U.S.S.R. 29, 38, 1952; 30, I6I, 1953; 31, 335, 1954.

(73) Su-Shu Huang, Ann. Astrophys. r6, 315, 1953.

(74) L. D. Chatterji, Proc. Nat. Acad. Sci. India, 17, 467, 195I and 18, 187, 1952; R. S. Kushwaha, Proc. Nat. Acad. Sci. India, 17, 323, 195I and 18, 461, 1952; R. S. Kushwaha and P. L. Bhatnagar, Proc. Nat. Acad. Sci. India, 18, 13, 1952; R. S. Kushwaha, Bull. Calcutta Math. Soc. 45, 75, 1953; C. Prasad, Proc. Nat. Acad. Sci. India, 19, 739, r953.

(75) G. Gjellestad, $A p . J$. 119, I4, I954 and 120, 172, 1954.

(76) V. C. A. Ferraro, $A p . J$. 119, 407, 1954.

(77) G. Gjellestad, Ann. Astrophys. 15, 276, I952.

(78) V. C. A. Ferraro and D. J. Memory, Mon. Not. R. Astr. Soc. r12, 361, 1952.

(79) C. Plumpton and V. C. A. Ferraro, Mon. Not. R. Astr. Soc. 1r3, 647, 1953.

(80) T. G. Cowling, Mon. Not. R. Astr. Soc. 112, 527, 1952.

(8I) P. A. Sweet, Mon. Not. R. Astr. Soc. 114, 549, 1954.

(82) S. Chandrasekhar and E. Fermi, $A p . J$. x18, I I6, 1953.

(83) S. Chandrasekhar and D. N. Limber, $A p . J$. I19, Io, I954.

(84) E. Lyttkens, $A p . J$. II9, 4I3, I954.

(85) N. Stoyko, C.R. Acad. Sci. Paris, 235, I22, 1952.

(86) M. H. Wrubel, $A p . J$. х16, 291, 1952.

(87) F. Hoyle, $A p . J$. I18, 5 r3, 1953.

(88) A. I. Lebedinsky, in Problems of Cosmogony, 2, p. 5.

(89) V. A. Krat, Pulkovo Bulletin, no. 149, 1952; C.R. Acad. Sci. U.R.S.S. 95, 481, 1954

(9o) V. A. Ambartsumian, Lg. 293.

(9I) P. Ledoux, Ann. Astrophys. 14, 438, 1951.

(92) L. E. Gurevich and A. I. Lebedinsky, C.R. Acad. Sci. U.R.S.S. 83, 81 3, I952.

(93) P. P. Parenago, Astr. J. U.S.S.R. 28, 93, I95I.

(94) S. A. Kaplan, Astr. J. U.S.S.R. 30, 391, 1953.

(95) E. R. Mustel, Lg. 223.

(96) E. Schatzman, Ann. Astrophys. 15, 126, 1952.

(97) H. Bondi, Mon. Not. R. Astr. Soc. 112, 195, 1952.

(98) W. H. McCrea and K. N. Dodd, Mon. Not. R. Astr. Soc. 112, 205, 1952.

(99) K. N. Dodd, Mon. Not. R. Astr. Soc. 112, 374, 1952.

(100) W. H. McCrea, Mon. Not. R. Astr. Soc. Ir3, 162, 1953; in Vistas in Astronomy, ed. A. Beer (London: Pergamon Press) (in the Press).

(I01) E. Schatzman, Report of second conference on the dynamics of interstellar matter (Amsterdam, North-Holland Publishing Company, 1955) p. 193.

(I02) L. Mestel, Mon. Not. R. Astr. Soc. rI4, 437, 1954.

(103) B. Strömgren, $A$ str. $J \cdot \mathbf{5 7}, 65,1952$.

(104) J. L. Greenstein, Lg. 307.

(105) O. Struve, Lg. 236.

(I06) R. J. Tayler, $A p . J$. 120, 332, 1954.

(107) A. G. Masevich, Astr. J. U.S.S.R. 30, 508, 1953; Comm. Sternberg Astron. Inst. (in the Press).

(108) E. Schatzman, Ann. Astrophys. 17, I 52, 377, $3^{82}$ and 398, 1954.

(109) H. L. Johnson and A. R. Sandage, $A p . J$. 121, 216, 1954; Lg. 254.

(1 Io) V. C. Reddish, Observatory, 74, 68, I954; Mon. Not. R. Astr. Soc. I14, 583, 1954.

(I I I) F. Hoyle and M. Schwarzschild, $A p . J$. Suppl. no. I3, 1955.

(I I2) A. Reiz, $A p . J$. r20, 342, I954.

(1 13) L. I. Sedov, Publ. $4^{\text {th }}$ Cosmogonic Conference, 1954 (in the Press). 
Report of the meeting. I September 1955

President: Prof. T. G. Cowling.

SECRETARY: Dr P. Ledoux.

The President's report was submitted and approved. The form adopted for the report was agreed to be generally acceptable.

Dr Keller outlined his work on stellar opacities, which agreed satisfactorily with that of Zirin and Rulsrud. He regarded his results as accurate within 10\%. The President thanked Dr Keller for circulating to members of the commission mimeographed tables of the opacity for thirteen different mixtures, and also for his offer to make available to persons interested punched-card records of opacity for machine calculations giving details of absorption edges and other information. In reply to questions, Dr Keller pointed out limitations of his work as applied to mixtures with small proportions of hydrogen and helium.

In a discussion of results for special stars, Dr Schwarzschild stated that recent computations for the Sun showed that estimates of hydrogen and helium abundances were considerably affected by taking into account an external convection-zone.

A discussion on the use of electronic computers followed. Attention was drawn to work not mentioned in the President's report, carried on at Göttingen (Kiepenhahn), Bloomington (Wrubel) and Berkeley (Henyey). Henyey had been able to study problems of stellar evolution, including time-derivatives, in his equations.

Dr Schwarzschild described his latest work on evolutionary tracks of stars burning up their energy-sources. Mme Masevich pointed out that this mode of evolution implied very low masses for red giants. She also suggested that the starting-point of the evolutionary sequence might be among the sub-dwarfs rather than on the main sequence.

Dr Schwarzschild suggested that the study of stellar structure might give important indications of differences of chemical composition between Populations I and II, since the stellar radius is very sensitive to composition in the outer parts of the star.

Prof. McCrea pointed out that such conclusions could be misleading, since a relatively small amount of accretion might modify composition in the surface layers appreciably.

Dr M. G. Burbidge described recent work on the synthesis of heavy elements by neutron-capture.

Attention was drawn by several members to the desirability of further work on convection designed particularly to clear up uncertainties in the value of the mixing-length.

The commission adopted the following resolution:

If the U.S.S.R. proposal for the institution of a Commission on Cosmogony should not prove acceptable, Commission 35 should undertake to cover work on Cosmogony which refers to stars. 